\title{
PROPERTIES OF STANDARD MAPS
}

\author{
GARY M. HUCKABAY ${ }^{1}$
}

\begin{abstract}
Let $X$ and $Y$ be compact metric spaces. Let $S(X, Y)$ denote the collection of standard maps of $X$ onto $Y$. We establish that $S(C, Y)$ is a dense subset of $C(C, Y)$, where $C$ is the Cantor set. If $f$ is a standard map and $G(f, Y)\{A(f, Y)\}$ denotes the subgroup of $H(X)$ which preserves \{interchanges\} the point-inverses of $f$, then there is a continuous homomorphism of $A(f, Y)$ into $H(Y)$ with kernel $G(f, Y)$. We also show that $G(f, Y)$ and $A(f, Y)$ are closed subsets of $H(X)$.
\end{abstract}

1. Let $X$ and $Y$ be topological spaces. Define $H(X, Y)\{C(X, Y)\}$ to be the collection of homeomorphisms \{continuous maps of $X$ onto $Y$. If $X=Y$ we agree to write $H(X)\{C(X)\}$. If $f \in C(X, Y)$, then $G(f, Y)$, defined by $\{h \in H(X): f \circ h=f\}$, is a subgroup of $H(X)$. Let us say that $f$ is a standard map if and only if (1) $f$ is an identification and (2) $f(a)=f(b)$ implies there are sequences $x_{n} \in X$ and $h_{n} \in G(f, Y)$ satisfying $x_{n} \rightarrow a$ and $h_{n}\left(x_{n}\right) \rightarrow b$. Standard maps were first studied by A. Vobach [5]. In [5], Vobach showed that each compact metric space is the standard image of $C$, and the author [3], [4] has shown that each locally compact, separable (complete, separable) metric space is the standard image of $N \times C(P)$, where $N$ denotes the positive integers and $P$ the irrationals. The utility of these results is that the standard images of a given space are classified up to homeomorphism by the conjugacy classes of the group $G(f, Y)([5],[3]$ and [4]).

2. In the sequel all spaces are compact and metrizable. We endow all function spaces with the compact-open topology.

Notation. $S(X, Y)=\{f \in C(X, Y): f$ is a standard map $\}$. If $f \in C(X, Y)$ we shall denote the partition $\left\{f^{-1}(y): y \in Y\right\}$ of $X$ by $K(f)$.

Definition. Let $f \in C(X, Y)$. We define the group $A(f, Y)$ to be $\{h \in$ $H(X): D \in K(f)$ implies $h(D) \in K(f)\}$.

REMARK. If $D \in K(f)$ and $h \in G(f, Y)$, then $h(D)=D$.

THEOREM 1. $G(f, Y)$ is a normal subgroup of $A(f, Y)$ and there is a continuous homomorphism $\alpha$ which takes $A(f, Y)$ into $H(Y)$ with kernel $G(f, Y)$.

Proof. We establish the latter statement first. Let $h \in A(f, Y)$ and define

Received by the editors September 13, 1976.

AMS (MOS) subject classifications (1970). Primary 54H99; Secondary 54E45, 54C10.

'This material will appear in the author's doctoral dissertation under the direction of Paul F. Duvall, Jr. at Oklahoma State Universitv.

(1) American Mathematical Society 1977 
$\alpha(h)=f \circ h \circ f^{-1}$. By a well-known theorem (see [1, p. 123]) $\alpha(h)$ is continuous if $\alpha(h)$ is single-valued. But this is clearly true since $h$ preserves the fiber structure of $f$. Similarly, $[\alpha(h)]^{-1}$ is continuous and therefore $\alpha(h) \in H(Y)$.

$\alpha$ is continuous: Let $(F, U)$ be any subbasic open subset of $H(Y)$. Then $\alpha^{-1}((F, U))=\left(f^{-1}(F), f^{-1}(U)\right) \cap A(f, Y)$. Hence $\alpha$ is continuous.

$\alpha$ is a homomorphism: Let $h_{1}, h_{2} \in A(f, Y)$. Then $\alpha\left(h_{1} \circ h_{2}\right)=$ $f \circ h_{1} \circ h_{2} \circ f^{-1}=\left(f \circ h_{1} f^{-1}\right) \circ\left(f \circ h_{2} \circ f^{-1}\right)$ since $h_{1}$ and $h_{2}$ preserve the fiber structure of $f$. Thus, $\alpha\left(h_{1} \circ h_{2}\right)=\alpha\left(h_{1}\right) \circ \alpha\left(h_{2}\right)$ as required.

kernel $\alpha=G(f, Y)$ : Clearly, for each $h \in G(f, Y), \alpha(h)=1_{Y}$. If $\alpha(h)=$ $1_{Y}$, then, by definition, $f \circ h \circ f^{-1}=1_{Y}$. For each $y \in Y$ we have $f\left(h\left(f^{-1}(y)\right)\right)=y$ or, equivalently, $h\left(f^{-1}(y)\right)=f^{-1}(y)$. But $h \in A(f, Y)$ and hence $h\left(f^{-1}(y)\right)=f^{-1}(y)$. Therefore, $h \in G(f, Y)$ and $G(f, Y)=$ kernel $\alpha$. This in turn implies the normality of $G(f, Y)$ in $A(f, Y)$.

Since $Y$ is compact and metrizable the compact-open topology on $C(Y)$ coincides with the sup-metric topology. Moreover, the sup-metric $d^{+}$on $C(Y)$ is a complete metric.

THEOREM 2. $A(f, Y)$ and $G(f, Y)$ are closed subsets of $H(X)$.

Proof. To see that $A(f, Y)$ is closed we consider a limit point $h$ of $A(f, Y)$ and a sequence $h_{n} \in A(f, Y)$ such that $h_{n} \rightarrow h$. Let $\alpha: A(f, Y) \rightarrow H(Y) \subset$ $C(Y)$ be the continuous homomorphism defined in Theorem 1. We will establish that $\alpha\left(h_{n}\right)$ converges to some element $k$ of $H(Y)$ and that $\alpha(h)=k$.

Let $p$ and $p^{+}\left\{d\right.$ and $\left.d^{+}\right\}$be the complete metrics of $X$ and $C(X)\{Y$ and $C(Y)\}$, respectively. Let $\varepsilon>0$ be given. Since $f$ is uniformly continuous there is a $\delta>0$ such that $d(f(x), f(y))<\varepsilon$ for each $x$ and $y$ satisfying $p(x, y)<\delta$. Now $h_{n} \rightarrow h$. Therefore, there is an integer $M$ such that $n, m>M$ implies $p^{+}\left(h_{n}, h_{m}\right)<\delta$. Thus,

$$
d\left(\alpha\left(h_{n}\right)(y), \alpha\left(h_{m}\right)(y)\right)=d\left(f\left(h_{n}\left(f^{-1}(y)\right)\right), f\left(h_{m}\left(f^{-1}(y)\right)\right)\right)<\varepsilon
$$

provided $n, m>M, y$ arbitrary. Hence $\alpha\left(h_{n}\right)$ is a Cauchy sequence in the complete space $C(Y)$. Let $\alpha\left(h_{n}\right) \rightarrow k \in C(Y)$. Then $f \circ h_{n} \circ f^{-1} \rightarrow k$ and $f \circ h_{n} \rightarrow k \circ f$. Therefore, $f \circ h=k \circ f$. In a similar way $f \circ h_{n}^{-1} \rightarrow f \circ h^{-1}=$ $k^{\prime} \circ f, k^{\prime} \in C(Y)$. Hence $k \circ k^{\prime}=1_{Y}=k^{\prime} \circ k$. Thus $k \in H(Y) . f \circ h \circ f^{-1}$ $=k$ implies $h \in A(f, Y)$. We conclude $A(f, Y)$ is closed.

$G(f, Y)$ is the kernel of a continuous homomorphism and therefore is closed in $A(f, Y)$. But $A(f, Y)$ is closed in $H(X)$ and hence $G(f, Y)$ is closed in $H(X)$.

REMARK. The reader should note that Theorems 1 and 2 do not require the map $f$ associated with $G(f, Y)$ and $A(f, Y)$ to be a standard map. In fact, Theorems 1 and 2 are true when $f$ is any continuous map.

Let $f \in C(C, X), X$ a compact metric space. Clearly we always have the inclusion $G(f, X) \subset A(f, X)$. Since $A(f, X)$ describes a subgroup of $H(X)$, one might ask whether $A(f, X)$ describes anything other than $\left\{1_{X}\right\}$. In other words, is it true that $A(f, X) \neq G(f, X)$ ? 
R. D. Anderson (see [2, p. 12]) has established the following theorem: Let $X$ be a compact metric space and $h \in H(X)$. Then there is a Cantor set $C$, $f \in C(C, X)$ and $\alpha \in H(X)$ such that $f \circ \alpha=h \circ f$. This shows that $f$ can be chosen from $C(C, X)$ in such a way that $A(f, X) \neq G(f, X)$ when $h \neq 1_{X}$. Using these techniques we prove in [3] that $f$ can always be chosen from $S(C, X)$.

Our last theorem deals with the relationship of $S(C, X)$ to $C(C, X)$. Let us first establish the following two lemmas.

Lemma 1. Let $X$ be homogeneous and $f \in S(Y, Z)$. Then $f \circ \pi: X \times Y \rightarrow Z$ is standard where $\pi$ is the projection map on the $Y$-coordinate.

Proof. This follows immediately from the homogeneity of $X$ and the standardness of $f$. The proof appears in [3].

LEMMA 2. Let $h$ be a homeomorphism of a Cantor set $C_{1}$ onto a Cantor set $C_{2}$. If $f \in S\left(C_{2}, X\right)$, then $f \circ h \in S\left(C_{1}, X\right)$.

Proof. The proof appears in [6].

TheOREM 3. Let $M$ be a compact metric space and $C$ the standard "middlethirds" Cantor set. If $f \in C(C, M)$ and $p \in S(C, M)$, then there is a $q \in$ $S(C, C)$ such that $p \circ q \in S(C, M)$ and $d^{+}(f, p \circ q)<\varepsilon, \varepsilon$ some preassigned positive number, and $d^{+}$the sup-metric on $C(C, M)$.

Proof. Let $C_{j}:\{1 \leqslant j \leqslant n\}$ be a decomposition of $C$ satisfying the following conditions:

(1) Each $C_{i}$ is both open and closed,

(2) $C_{i} \cap C_{j}=\varnothing, i \neq j$, and

(3) diam $f\left(C_{j}\right)<\varepsilon$ for each $i$.

Define $E_{j}=p^{-1}\left(f\left(C_{j}\right)\right)$ and $D_{j}=C \times E_{j} \times\{1 / j\}$. Let $\mathscr{D}=\cup\left\{D_{j}: 1 \leqslant j\right.$ $\leqslant n\}$ and note $D_{j} \simeq \mathscr{D} \simeq C$. If $h \in G(p, M)$, then $h\left(E_{j}\right)=E_{j}$ for all $j$. Construct $\alpha \in H(C, D)$ such that $\alpha\left(C_{j}\right)=D_{j}$ and define $q \in C(C, C)$ by $q=\pi \circ \alpha$ where $\pi$ is the projection map on the $E_{j}$-coordinate, $\pi \in S(\mathscr{D}, C)$. If $\pi\left(c_{1}, e_{1}, 1 / j_{1}\right)=\pi\left(c_{2}, e_{2}, 1 / j_{2}\right)$, then $e_{1}=e_{2}$ by definition of $\pi$. Let $x_{n}=$ $\left(c_{1}, e_{1}, 1 / j_{1}\right)$ for each $n$ and choose $h \in H(C), k \in H(\{1 / 1,1 / 2, \ldots, 1 / n\})$ such that $h\left(c_{1}\right)=c_{2}, k\left(1 / j_{1}\right)=1 / j_{2}$, respectively. Then $\left(h, 1_{C}, k\right) \in G(\pi, C)$ and $\left(h, 1_{C}, k\right)\left(x_{n}\right) \rightarrow\left(c_{2}, e_{2}, 1 / j_{2}\right)$ (equals in fact) as required. In view of Lemma 2, $\pi \circ \alpha \in S(C, C)$. Define $g \in C(C, M)$ by $g=p \circ q=(p \circ \pi) \circ \alpha$. By Lemma 2, $g \in S(C, M)$ if $p \circ \pi \in S(\mathcal{Q}), M)$. But $p \circ \pi \in S(\mathcal{Q}), M)$ by Lemma 1 . Hence $g \in S(C, M)$.

Let $x \in C$ and $x \in C_{j}$ for some $j$. Consider $g(x)=p(\pi(\alpha(x)))$. Then $\alpha(x) \in D_{j}$ and $\pi(\alpha(x)) \in E_{j}$. Therefore, $p(\pi(\alpha(x))) \in f\left(C_{j}\right)$ which yields $d^{+}(f, p \circ q)<\varepsilon$.

COROllary 1. $S(C, M)$ is a dense subset of $C(C, M)$.

REMARK. Note that Theorem 3 actually proves that $\{p \circ S(C, C)\} \cap$ 
$S(C, M)$ is a dense subset of $C(C, M)$ for each $p \in S(C, M)$.

\section{REFERENCES}

1. J. Dugundji, Topology, Allyn and Bacon, Boston, Mass., 1966. MR 33 \# 1824.

2. W. Gottschalk and J. Auslander (editors), Topological dynamics, Benjamin, New York, 1968. MR 38 \#2762.

3. G. Huckabay, On the classification of locally compact, separable metric spaces, Fund. Math. (to appear).

4. Standard maps and the classification of topological spaces, $\mathrm{Ph} . \mathrm{D}$. thesis, Oklahoma State University.

5. A. Vobach, On subgroups of the homeomorphism group of the Cantor set, Fund. Math. 60 (1967), 47-52. MR 36 \#2108.

6. , A theorem on homeomorphism groups and products of spaces, Bull. Austral. Math. Soc. 1 (1969), 137-141. MR 39 \#7586.

Department of Mathematics, Cameron University, Lawton, Oklahoma 73501 (Current address)

Department of Mathematics, OKlahoma State University, Stillwater, Oklahoma 74074 